\title{
Association of HLA-DRB1 and -DQB1 Alleles with Susceptibility to IgA Nephropathy in Korean Patients
}

\author{
Ji Won In (1), M.D. ${ }^{1}$, Kiwook Jung (i), M.D. ${ }^{2}$, Sue Shin (1), M.D., Ph.D. ${ }^{2}$, Kyoung Un Park (i), M.D., Ph.D. ${ }^{2}$, \\ Hajeong Lee $\mathbb{1}$, M.D., Ph.D. ${ }^{3}$, and Eun Young Song $\mathbb{~} \bullet$, M.D., Ph.D. ${ }^{2}$ \\ ${ }^{1}$ Department of Laboratory Medicine, Seoul Medical Center, Seoul, Korea; ${ }^{2}$ Department of Laboratory Medicine, Seoul National University College of \\ Medicine, Seoul, Korea; ${ }^{3}$ Department of Internal Medicine, Seoul National University Hospital, Seoul, Korea
}

Background: Associations between IgA nephropathy (IgAN) and HLA-DRB1 and -DQB1 alleles have been reported in several ethnic groups. We investigated the association of HLADRB1 and -DQB1 alleles with the predisposition for IgAN and disease progression to endstage kidney disease (ESKD) in Korean patients.

Methods: We analyzed HLA-DRB1 and -DQB1 genotypes in 399 IgAN patients between January 2000 and January 2019 using a LIFECODES sequence-specific oligonucleotide (SSO) typing kit (Immucor, Stamford, CT, USA) or a LABType SSO Typing Test (One Lambda, Canoga Park, CA, USA). Alleles with a significant difference in two-digit resolution were further analyzed using in-house sequence-based typing and sequence-specific primer PCR. As controls, 613 healthy hematopoietic stem cell donors were included. Kidney survival was analyzed in 281 IgAN patients with available clinical and laboratory data using Cox regression analysis. Where needed, $P$-values were adjusted using Bonferroni correction.

Results: The allele frequencies of HLA-DRB ${ }^{*} 04: 05$ (corrected $P[P C]<0.001$ ), -DQB1 ${ }^{*} 04: 01(P C=0.048)$, and $-\mathrm{DQB1}{ }^{*} 03: 02(P C=0.021)$ were significantly higher in IgAN patients than in controls, whereas those of HLA-DRB1*07:01, -DRB1*15:01, -DQB1*02:02, and $-\mathrm{DQB} 1{ }^{*} 06: 02(P C<0.001$ for all) were significantly lower in IgAN patients than in controls. The allele frequency of $\mathrm{HLA}-\mathrm{DQB1}{ }^{*} 05: 03(P C=0.016)$ was significantly lower in the ESKD group than in the non-ESKD group; however, there was no significant difference for ESKD progression between these groups.

Conclusions: We report novel associations of HLA-DRB1*15:01, DQB1*02:02, -DQB1*03:02, and -DQB1*04:01 with IgAN. Further studies of HLA alleles associated with IgAN progression in a larger cohort and in various ethnic groups are needed.

Key Words: IgA nephropathy, Human leukocyte antigen, DRB1, DQB1, Association, Endstage kidney disease, Disease progression
Received: December 1, 2020

Revision received: December 28, 2020

Accepted: July 21, 2021

\section{Corresponding author:}

Eun Young Song, M.D., Ph.D.

Department of Laboratory Medicine, Seoul National University College of Medicine,

101 Daehak-ro, Jongno-gu, Seoul 03080, Korea

Tel: +82-2-2072-0197

Fax: +82-2-747-0359

E-mail: eysong1@snu.ac.kr

\section{Co-corresponding author:}

Hajeong Lee, M.D., Ph.D.

Department of Internal Medicine, Seoul National University College of Medicine, 101 Daehak-ro, Jongno-gu, Seoul 03080, Korea

Tel: +82-2-2072-4905

E-mail: mdhjlee@gmail.com

\section{(c) $\underset{\mathrm{BY}}{\mathrm{i}} \mathrm{NC}$}

(C) Korean Society for Laboratory Medicine This is an Open Access article distributed under the terms of the Creative Commons Attribution Non-Commercial License (https://creativecommons.org/licenses/by-nc/4.0) which permits unrestricted non-commercial use, distribution, and reproduction in any medium, provided the original work is properly cited.

\section{INTRODUCTION}

IgA nephropathy (IgAN) is the most frequent form of primary glomerulonephritis exhibiting geographic and ethnic differences, and is prevalent in Asian countries [1, 2]. In many patients, it gradually progresses to end-stage kidney disease (ESKD). IgAN patients in the Asia-Pacific region particularly have a high risk of disease progression [3]. Familial manifestations, along with autoimmune abnormalities, are frequently reported in IgAN patients, which has led to studies on IgAN immunopathogenesis and genetic pathogenesis [4]. Since the 1980s, human leukocyte antigen (HLA) molecules related to $T$ lymphocyte activity have been shown to be associated with genetic susceptibility to IgAN with varying results [5-10]. In several genome-wide asso- 
ciation studies (GWASs), single-nucleotide variations (SNVs) related to the HLA-DRB1 and -DQB1 loci have been reported to have a strong association with IgAN [11-13]. In Korea, a study on 139 IgAN patients reported associations between IgAN and HLA alleles [14]. The allele frequencies of HLA-DQB1*03:02 and $-\mathrm{DQB1}{ }^{*} 05: 03$ were higher in pediatric and adult IgAN patients with ESKD than in pediatric IgAN patients with normal kidney function, but the HLA-DR allele frequency showed no difference between these groups. However, a GWAS in 188 Korean IgAN patients did not show significant associations between HLA loci-related SNVs and IgAN [15]. With the aim to expand the knowledge of associations between HLA alleles and IgAN in a larger cohort of Korean patients regardless of age, we analyzed HLA-DRB1 and -DQB1 alleles in 399 Korean IgAN patients and investigated the association between these HLA alleles and IgAN susceptibility and prognosis.

\section{MATERIALS AND METHODS}

\section{Study population and clinical data}

Among the Korean patients diagnosed as having IgAN at Seoul National University Hospital (SNUH, Seoul, Korea) between January 1, 2000 and January 1, 2019, 268 IgAN patients with ESKD and 131 patients with IgAN that did not progress to ESKD were enrolled in this study (Fig. 1). Among the 131 patients, 57 patients did not progress to ESKD after more than 10 years of follow up and 74 patients did not progress to ESKD after less than 10 years of follow up. These patients were diagnosed as having IgAN by kidney biopsy at SNUH or by slide review of biopsy performed outside SNUH. Patients with clinical or laboratory ev- idence of systemic lupus erythematosus, liver cirrhosis, and Henoch-Schönlein nephritis at the time of diagnosis were excluded. ESKD was defined as the initiation of permanent dialysis or kidney transplantation. Patient data were obtained through retrospective medical record reviews. The observation period ended on December 31, 2019, and data from patients who missed follow-up or with preserved kidney function were censored on the day the last kidney function was confirmed. The Institutional Review Board (IRB) of SNUH (IRB No. 1911-009-1074) approved this study and exempted the requirement of an informed consent.

Kidney survival was analyzed in 281 IgAN patients (153 with ESKD and 128 not progressed to ESKD) for whom all clinical and laboratory data were available at the time of initial biopsy (Fig. 1). For IgAN patients, clinical and laboratory data including sex, age at the time of biopsy, blood pressure, plasma $\mathrm{Hb}$, serum creatinine $(\mathrm{Cr})$ level, estimated glomerular filtration rate (eGFR), serum albumin level, serum lgA level, gross hematuria, microscopic hematuria, and 24-hour urinary protein, were obtained. Hypertension was defined as systolic blood pressure $\geq 140 \mathrm{~mm} \mathrm{Hg}$, diastolic blood pressure $\geq 90 \mathrm{~mm} \mathrm{Hg}$, or undergoing treatment for hypertension. The eGFR was calculated using the modification of diet in renal disease formula. For each patient, the duration from diagnosis to onset of ESKD was noted. The risk of IgAN progression to ESKD was analyzed based on the clinical and laboratory data and HLA allele types. When analyzing risk factors, the 24-hour urinary protein was classified based on a threshold of $1 \mathrm{~g} /$ day.

The characteristics of IgAN patients with all available clinical and laboratory data at the time of kidney biopsy are presented

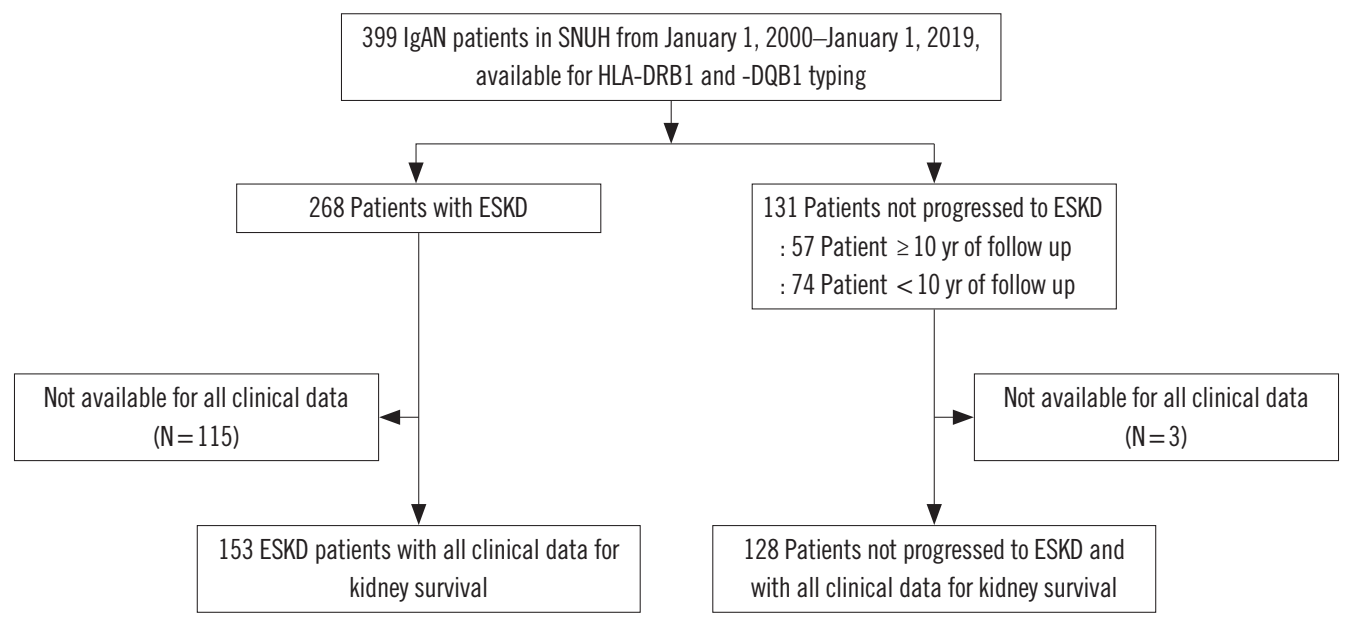

Fig. 1. Study population.

Abbreviations: IgAN, IgA nephropathy; SNUH, Seoul National University Hospital; ESKD, end-stage kidney disease. 
Table 1. Clinical characteristics of IgAN patients with all available clinical data at the time of kidney biopsy $(\mathrm{N}=281)$

\begin{tabular}{|c|c|c|c|c|}
\hline Characteristics & $\begin{array}{c}\text { IgAN patients with all clinical } \\
\text { data }(\mathrm{N}=281)\end{array}$ & $\begin{array}{l}\text { ESKD } \\
(N=153)\end{array}$ & $\begin{array}{l}\text { not progressed to ESKD } \\
(\mathrm{N}=128)\end{array}$ & $p^{*}$ \\
\hline Age (yr) & $37(28-47)$ & $36(28-47)$ & $38(28-48)$ & 0.340 \\
\hline Sex (male, \%) & $136(48.4)$ & $82(53.6)$ & $54(42.2)$ & 0.057 \\
\hline Hypertension (\%) & $176(62.6)$ & $109(71.2)$ & $67(52.3)$ & 0.001 \\
\hline Plasma Hb (g/L) & $132(115-145)$ & $126(108-141)$ & 136 (122-149) & $<0.001$ \\
\hline Serum $\operatorname{Cr}(\mu \mathrm{mol} / \mathrm{L})$ & $144.92(88.40-159.12)$ & $150.28(114.92-240.89)$ & $88.40(74.48-106.08)$ & $<0.001$ \\
\hline eGFR (mL/min/1.73 m²) & $58.8(38.9-81.9)$ & 41.6 (23.7-60.8) & 77.45 (60.85-92.58) & $<0.001$ \\
\hline Serum albumin (g/L) & $39(36-43)$ & $38(34-40)$ & $42(39-44)$ & $<0.001$ \\
\hline Serum IgA (g/L) & $3.16(2.50-4.10)$ & $3.15(2.49-4.10)$ & $3.20(2.52-4.10)$ & 0.688 \\
\hline Gross hematuria (\%) & $62(22.1)$ & $27(17.6)$ & $35(27.3)$ & 0.051 \\
\hline Microscopic hematuria (\%) & $253(90.0)$ & $137(89.5)$ & $116(90.6)$ & 0.763 \\
\hline 24-hr urinary protein ( $\mathrm{g} /$ day) & $1.6(0.8-2.9)$ & $2.3(1.5-4.0)$ & $0.9(0.5-1.5)$ & $<0.001$ \\
\hline
\end{tabular}

Values are presented as median (interquartile range) or number (\%).

*ESKD vs. not progressed to ESKD

Abbreviations: IgAN, IgA nephropathy; ESKD, end-stage kidney disease; $\mathrm{Cr}$, creatinine; eGFR, estimated glomerular filtration rate.

in Table 1. There were significant differences in blood pressure, plasma $\mathrm{Hb}$ level, serum $\mathrm{Cr}$ level, eGFR, serum albumin level, and 24-hour urinary protein between ESKD patients and those who not progressed to ESKD.

To calculate the HLA allele frequency in the controls, we used data from 613 healthy Korean hematopoietic stem cell donors collected between January 2006 and July 2014 and analyzed in previous study [16].

\section{HLA-DRB1 and -DQB1 genotyping}

HLA-DRB1 and -DQB1 typing of 399 IgAN patients were performed with DNA samples preserved at $-70^{\circ} \mathrm{C}(\mathrm{N}=209)$ or newly extracted from the preserved EDTA blood at $-70^{\circ} \mathrm{C}(\mathrm{N}=190)$ provided by the Biobank of Seoul National University Hospital. Genomic DNA was extracted by using EZ1 DNA Blood $350 \mu \mathrm{L}$ Kit (Qiagen, Hilden, Germany). HLA-DRB1 and -DQB1 were typed by sequence-specific oligonucleotide (SSO) typing using the LIFECODES SSO Typing Kit (Immucor, Stamford, CT, USA) or the LABType SSO Typing Test (One Lambda, Canoga Park, CA, USA), according to the manufacturers' instructions. HLADRB1 alleles showing a frequency difference $(P<0.05)$ at twodigit resolution were further analyzed at four-digit resolution by sequence-based typing (SBT) or sequence-specific primer PCR (PCR-SSP). For HLA-DQB1 (except HLA-DQB1*04) typing, fourdigit resolution was assigned by SSO typing using the two abovementioned kits $[17,18]$. HLA-DQB1*04 subtyping was confirmed by SBT.

HLA-DRB $1{ }^{*} 02$, -DRB $1{ }^{*} 04$, and -DQB $1{ }^{*} 04$ subtyping was performed using PCR-SSP or SBT, according to the reported methods and primer design [19, 20]. The PCR conditions were as follows: initial denaturation at $94^{\circ} \mathrm{C}$ for 5 minutes; 35 cycles of denaturation at $94^{\circ} \mathrm{C}$ for 30 seconds, annealing at $62^{\circ} \mathrm{C}$ for 30 seconds, and elongation at $72^{\circ} \mathrm{C}$ for 60 seconds; and a final elongation step at $72^{\circ} \mathrm{C}$ for 7 minutes. For SBT, each ExoSAP-IT (Affymetrix, Santa Clara, CA, USA) PCR product was sequenced using the primers used for PCR, deionized water, and BigDye Terminator Ready Reaction Mix (Life Technologies, Grand Island, NY, USA), using an initial denaturation step at $96^{\circ} \mathrm{C}$ for 1 minute, 30 cycles of denaturation at $96^{\circ} \mathrm{C}$ for 10 seconds, annealing at $50^{\circ} \mathrm{C}$ for 5 seconds, and elongation at $60^{\circ} \mathrm{C}$ for 4 minutes. After ethanol precipitation to remove the unbound terminator, the product was heated for 4 minutes and analyzed on an ABI 3730XL DNA Analyzer (Applied Biosystems, Foster City, CA, USA); electropherograms were analyzed using Chromas Lite 2.1.1 (Technelysium, South Brisbane, Australia).

\section{Statistical analysis}

All data were analyzed using SPSS software (version 26.0, IBM Corp., Armonk, NY, USA). Continuous variables (age, plasma $\mathrm{Hb}$, serum $\mathrm{Cr}$, eGFR, serum albumin, serum IgA, 24-hours urinary protein) are presented as medians and interquartile ranges, and categorical variables (sex, hypertension, gross hematuria, microscopic hematuria) are shown as frequencies and percentages. Clinical characteristics, laboratory data, and HLA allele frequencies were compared between the groups using the MannWhitney test, chi-square test or Fisher's exact test, as appropri- 
In JW, et al.

HLA allele association with IgAN in Koreans

Table 2. HLA-DRB1 and -DQB1 allele frequencies in IgAN patients and controls

\begin{tabular}{|c|c|c|c|c|c|c|c|c|c|}
\hline \multirow{2}{*}{ Allele } & \multirow{2}{*}{ Subgroup } & \multicolumn{2}{|c|}{$\operatorname{IgAN}(N=399 \times 2)$} & \multicolumn{2}{|c|}{ Control $(N=613 \times 2)$} & \multirow{2}{*}{$P$} & \multirow{2}{*}{$P c$} & \multirow{2}{*}{$\mathrm{OR}$} & \multirow{2}{*}{$95 \% \mathrm{Cl}$} \\
\hline & & $\mathrm{N}$ & $\%$ & $\mathrm{~N}$ & $\%$ & & & & \\
\hline DRB1*01 & & 42 & 5.26 & 79 & 6.44 & 0.274 & & & \\
\hline $\mathrm{DRB} 1$ *03 & & 15 & 1.88 & 26 & 2.12 & 0.707 & & & \\
\hline \multirow[t]{9}{*}{$\mathrm{DRB} 1$ *04 } & & 242 & 30.33 & 259 & 21.13 & $<0.001$ & $<0.001$ & 1.63 & $1.33-1.99$ \\
\hline & $\mathrm{DRB} 1 * 04: 01$ & 4 & 0.50 & 12 & 0.98 & & & & \\
\hline & DRB1*04:03 & 30 & 3.76 & 38 & 3.10 & & & & \\
\hline & DRB1*04:04 & 13 & 1.63 & 24 & 1.96 & & & & \\
\hline & DRB1*04:05 & 119 & 14.91 & 111 & 9.05 & $<0.001$ & $<0.001$ & 1.76 & $1.34-2.32$ \\
\hline & DRB1*04:06 & 56 & 7.02 & 51 & 4.16 & 0.005 & 0.150 & 1.74 & $1.18-2.57$ \\
\hline & DRB1*04:07 & 1 & 0.13 & 6 & 0.49 & & & & \\
\hline & DRB1*04:08 & 1 & 0.13 & 0 & 0.00 & & & & \\
\hline & $\mathrm{DRB} 1 * 04: 10$ & 18 & 2.26 & 17 & 1.39 & & & & \\
\hline $\mathrm{DRB} 1 * 07$ & $\mathrm{DRB} 1 * 07: 01$ & 26 & 3.26 & 85 & 6.93 & $<0.001$ & $<0.001$ & 0.45 & $0.29-0.71$ \\
\hline $\mathrm{DRB} 1 * 08$ & & 83 & 10.40 & 105 & 8.56 & 0.164 & & & \\
\hline DRB1*09 & & 57 & 7.14 & 116 & 9.46 & 0.068 & & & \\
\hline $\mathrm{DRB} 1 * 10$ & & 23 & 2.88 & 24 & 1.96 & 0.177 & & & \\
\hline DRB1*11 & & 36 & 4.51 & 48 & 3.92 & 0.511 & & & \\
\hline DRB1*12 & & 54 & 6.77 & 93 & 7.59 & 0.488 & & & \\
\hline DRB1*13 & & 84 & 10.53 & 135 & 11.01 & 0.731 & & & \\
\hline DRB1*14 & & 83 & 10.40 & 99 & 8.08 & 0.074 & & & \\
\hline \multirow[t]{3}{*}{$\mathrm{DRB} 1 * 15$} & & 46 & 5.76 & 147 & 11.99 & $<0.001$ & $<0.001$ & 0.45 & $0.32-0.63$ \\
\hline & DRB1*15:01 & 34 & 4.26 & 101 & 8.24 & $<0.001$ & $<0.001$ & 0.50 & $0.33-0.74$ \\
\hline & DRB1*15:02 & 12 & 1.50 & 46 & 3.75 & 0.003 & 0.090 & 0.39 & $0.21-0.74$ \\
\hline $\mathrm{DRB} 1 * 16$ & & 7 & 0.88 & 10 & 0.82 & 1.000 & & & \\
\hline \multirow[t]{3}{*}{$\mathrm{DQB} 1 * 02$} & & 40 & 5.01 & 108 & 8.81 & 0.001 & 0.007 & 0.55 & $0.38-0.79$ \\
\hline & $\mathrm{DQB} 1 * 02: 01$ & 15 & 1.88 & 26 & 2.12 & & & & \\
\hline & $\mathrm{DQB1}{ }^{*} 02: 02$ & 25 & 3.13 & 82 & 6.69 & $<0.001$ & $<0.001$ & 0.45 & $0.29-0.71$ \\
\hline \multirow[t]{3}{*}{$\mathrm{DQB1} 1 * 04$} & & 143 & 17.92 & 156 & 12.72 & 0.001 & 0.007 & 1.50 & $1.17-1.92$ \\
\hline & $\mathrm{DQB1}{ }^{*} 04: 01$ & 103 & 12.91 & 108 & 8.81 & 0.003 & 0.048 & 1.53 & $1.15-2.04$ \\
\hline & $\mathrm{DQB1} 1 * 04: 02$ & 40 & 5.01 & 48 & 3.92 & & & & \\
\hline $\mathrm{DQB} 1 * 05$ & & 139 & 17.42 & 191 & 15.58 & 0.274 & & & \\
\hline \multirow[t]{9}{*}{$\mathrm{DQB} 1 * 06$} & & 189 & 23.68 & 344 & 28.06 & 0.029 & 0.203 & 0.80 & $0.65-0.98$ \\
\hline & $\mathrm{DQB} 1 * 06: 01$ & 74 & 9.27 & 115 & 9.38 & & & & \\
\hline & $\mathrm{DQB1} 1 * 06: 02$ & 30 & 3.76 & 95 & 7.75 & $<0.001$ & $<0.001$ & 0.47 & $0.31-0.71$ \\
\hline & $\mathrm{DQB} 1 * 06: 03$ & 6 & 0.75 & 20 & 1.63 & & & & \\
\hline & $\mathrm{DQB} 1 * 06: 04$ & 58 & 7.27 & 62 & 5.06 & 0.040 & 0.640 & 1.47 & $1.02-2.13$ \\
\hline & $\mathrm{DQB1}{ }^{* 06: 09}$ & 21 & 2.63 & 52 & 4.24 & & & & \\
\hline & DQ7 (DQB1*03:01) & 108 & 13.53 & 172 & 14.03 & 0.752 & & & \\
\hline & DQ8 (DQB1*03:02) & 111 & 13.91 & 118 & 9.62 & 0.003 & 0.021 & 1.52 & $1.15-2.00$ \\
\hline & DQ9 (DQB1*03:03) & 68 & 8.52 & 137 & 11.17 & 0.053 & & & \\
\hline
\end{tabular}

Abbreviations: IgAN, IgA nephropathy; $P C$, corrected $P$-value; OR, odds ratio; $\mathrm{Cl}$, confidence interval. 
ate. $P$-values were adjusted using the Bonferroni correction, i.e., by multiplying the $P$-value by the number of comparisons, considering the total number of alleles in patients and controls that could be obtained at each resolution (corrected $P[P C]$ for twodigit resolution: 13 for HLA-DRB1 and 7 for HLA-DQB1; PC for four-digit resolution: 30 for HLA-DRB1 and 16 for HLA-DQB1). Odds ratio (OR) was calculated along with 95\% confidence interval $(\mathrm{Cl})$. Univariate and multivariate Cox regression analysis were used to analyze risk factors associated with ESKD. $P C<0.05$ in the allele frequency analysis and $P<0.05$ in the survival analysis were considered statistically significant.

\section{RESULTS}

HLA-DRB1 allele frequencies in IgAN patients

HLA-DRB1 allele frequencies for the 399 IgAN patients and con- trols are shown in Table 2 . The frequency of the HLA-DRB ${ }^{*} 04$ allele was significantly higher in the patients than in the controls. In the patients, the allele frequencies of HLA-DRB1 ${ }^{*} 07$ (with one allelic subgroup in Koreans, DRB1*07:01) and -DRB1*15 were significantly lower than those in the controls. Since nearly all HLA-DRB $1{ }^{*} 07$ alleles in Koreans are HLA-DRB1 ${ }^{*} 07: 01$, no additional typing was performed [21]. When analyzed at the four-digit resolution, HLA-DRB $1{ }^{*}$ 04:05 showed a significantly higher frequency in the IgAN patients than in the controls. HLADRB $1{ }^{*}$ 04:06 tended to be slightly, albeit not significantly, increased in the IgAN patients compared to the controls. The allele frequency of HLA-DRB1*15:01 was significantly lower in the IgAN patients than in the controls, whereas the allele frequency of HLA-DRB1*15:02 tended to be lower, albeit not significantly, in the patients than in the controls.

Table 3. Comparison of HLA-DRB1 and -DQB1 allele frequencies between the ESKD and non-ESKD groups*

\begin{tabular}{|c|c|c|c|c|c|c|c|c|}
\hline \multirow{2}{*}{ Allele } & \multicolumn{2}{|c|}{ ESKD $(N=268 \times 2)$} & \multicolumn{2}{|c|}{ Non-ESKD $(\mathrm{N}=57 \times 2)$} & \multirow{2}{*}{$P$} & \multirow{2}{*}{$P c$} & \multirow{2}{*}{ OR } & \multirow{2}{*}{$95 \% \mathrm{Cl}$} \\
\hline & $\mathrm{N}$ & $\%$ & $\mathrm{~N}$ & $\%$ & & & & \\
\hline \multicolumn{9}{|l|}{ DRB1 } \\
\hline $\mathrm{DRB} 1 * 01$ & 24 & 4.48 & 4 & 3.51 & 0.802 & & & \\
\hline $\mathrm{DRB} 1 * 03$ & 9 & 1.68 & 5 & 4.39 & 0.081 & & & \\
\hline $\mathrm{DRB} 1 * 04$ & 171 & 31.90 & 24 & 21.05 & 0.022 & 0.286 & 1.76 & $1.08-2.86$ \\
\hline $\mathrm{DRB} 1 * 07$ & 19 & 3.54 & 2 & 1.75 & 0.557 & & & \\
\hline $\mathrm{DRB} 1 * 08$ & 55 & 10.26 & 12 & 10.53 & 0.933 & & & \\
\hline $\mathrm{DRB} 1 * 09$ & 41 & 7.65 & 5 & 4.39 & 0.217 & & & \\
\hline $\mathrm{DRB} 1 * 10$ & 18 & 3.36 & 3 & 2.63 & 1.000 & & & \\
\hline $\mathrm{DRB} 1 * 11$ & 23 & 4.29 & 7 & 6.14 & 0.393 & & & \\
\hline $\mathrm{DRB} 1 * 12$ & 35 & 6.53 & 13 & 11.40 & 0.071 & & & \\
\hline DRB1*13 & 58 & 10.82 & 14 & 12.28 & 0.652 & & & \\
\hline $\mathrm{DRB} 1 * 14$ & 52 & 9.70 & 18 & 15.79 & 0.057 & & & \\
\hline DRB1*15 & 27 & 5.04 & 6 & 5.26 & 0.921 & & & \\
\hline $\mathrm{DRB} 1 * 16$ & 4 & 0.75 & 1 & 0.88 & 1.000 & & & \\
\hline \multicolumn{9}{|l|}{ DQB1 } \\
\hline $\mathrm{DQB1} 1 * 02$ & 26 & 4.85 & 7 & 6.14 & 0.569 & & & \\
\hline $\mathrm{DQB1} 1 * 04$ & 103 & 19.22 & 13 & 11.40 & 0.048 & 0.336 & 1.85 & $1.00-3.42$ \\
\hline $\mathrm{DQB1}{ }^{*} 05$ & 86 & 16.04 & 23 & 20.18 & 0.284 & & & \\
\hline DQB1*05:03 & 24 & 4.48 & 14 & 12.28 & 0.001 & 0.016 & 0.34 & $0.17-0.67$ \\
\hline $\mathrm{DQB} 1{ }^{*} 06$ & 128 & 23.88 & 29 & 25.44 & 0.724 & & & \\
\hline DQ7 (DQB1*03:01) & 68 & 12.69 & 21 & 18.42 & 0.106 & & & \\
\hline DQ8 (DQB1*03:02) & 74 & 13.81 & 14 & 12.28 & 0.666 & & & \\
\hline DQ9 (DQB1*03:03) & 51 & 9.51 & 7 & 6.14 & 0.251 & & & \\
\hline
\end{tabular}

*Not progressed to ESKD even after 10 years from diagnosis

Abbreviations: ESKD, end-stage kidney disease; $P C$, corrected $P$-value; OR, odds ratio; $\mathrm{Cl}$, confidence interval. 
HLA-DQB1 allele frequencies in IgAN patients

The HLA-DQB1 allele frequencies for the 399 IgAN patients and controls are shown in Table 2. The allele frequencies of HLADQB1*04 and -DQ8 (DQB1*03:02) were significantly higher in the IgAN patients than in the controls. The allele frequency of HLA-DQB1 ${ }^{*} 02$ was significantly lower in the patients than in the controls. In the four-digit resolution analysis, the frequency of HLA-DQB ${ }^{*} 04: 01$ was significantly higher in the IgAN patients than in the controls. In the IgAN patients, the allele frequency of HLA-DQB1*02:02 was significantly lower than that in the controls. Among the HLA-DQB1*06 allele subgroups, the HLA-DQB1 *06:02 allele showed a significantly lower frequency in the IgAN patients than in the controls. Conversely, the HLA-DQB1 ${ }^{*} 06: 04$ allele frequency was slightly, albeit not significantly, higher in the IgAN patients than in the controls.

\section{Comparison of HLA-DRB1 and -DQB1 allele frequencies between ESKD and non-ESKD groups}

HLA-DRB1 and -DQB1 allele frequencies were compared between 268 IgAN patients that progressed to ESKD (ESKD group) and 57 IgAN patients with preserved kidney function even after 10 years from the time of diagnosis (non-ESKD group) (Table 3) (The remaining 74 patients not progressed to ESKD after less than 10 years of follow up were excluded for comparison because the observation period was not long enough). In the ESKD group, the allele frequencies of HLA-DRB1 ${ }^{*} 04$ and $-\mathrm{DQB} 1{ }^{*} 04$ were slightly, albeit not significantly, higher than those in the non-ESKD group. In the four-digit resolution analysis of HLA-DQB1, the allele frequency of $\mathrm{HLA}-\mathrm{DQB} 1{ }^{*} 05: 03$ was significantly lower in the ESKD group than in the non-ESKD group.

\section{Kidney survival analysis and prognostic factors for ESKD}

Kidney survival analysis results are shown in Table 4. The duration of disease progression to ESKD from the time of diagnosis was 57 (25-101) months. In univariate Cox proportional hazard models, hypertension, plasma $\mathrm{Hb}$, serum $\mathrm{Cr}$, eGFR, serum albumin, and 24-hour urinary protein $>1 \mathrm{~g} /$ day were associated with an increased risk of ESKD. Multivariate Cox regression analysis with the variables that were found to be significant in the univariate Cox regression analysis showed that serum $\mathrm{Cr}$ levels $(P<0.001$, hazard ratio $(\mathrm{HR})=1.25,95 \% \mathrm{Cl}=1.11-1.42)$, eGFR $(P<0.001, \mathrm{HR}=0.98,95 \% \mathrm{Cl}=0.97-0.99)$, and 24-hour urinary protein $>1 \mathrm{~g} /$ day $(P<0.001, \mathrm{HR}=2.88,95 \% \mathrm{Cl}=1.83$ 4.55) were independent risk factors for ESKD progression. However, HLA alleles were not significant risk factors for ESKD progression.

\section{DISCUSSION}

Since 1978, when it was reported that a brother who donated a

Table 4. Univariate and multivariate Cox regression analyses of prognostic factors of IgAN progression to ESKD $(\mathrm{N}=281)$

\begin{tabular}{|c|c|c|c|c|c|c|}
\hline \multirow{2}{*}{ Prognostic factors } & \multicolumn{3}{|c|}{ Cox regression univariate analysis } & \multicolumn{3}{|c|}{ Cox regression multivariate analysis } \\
\hline & HR & $95 \% \mathrm{Cl}$ & $P$ & $\mathrm{HR}$ & $95 \% \mathrm{Cl}$ & $P$ \\
\hline Age at biopsy (yr) & & & 0.498 & & & \\
\hline Sex (female) & & & 0.141 & & & \\
\hline Hypertension (+) & 2.07 & $1.45-2.94$ & $<0.001$ & & & \\
\hline Plasma Hb, per $1 \mathrm{~g} / \mathrm{L}$ greater & 0.77 & $0.71-0.83$ & $<0.001$ & & & \\
\hline Serum Cr, per $1 \mu \mathrm{mol} / \mathrm{L}$ greater & 1.57 & $1.44-1.70$ & $<0.001$ & 1.25 & $1.11-1.42$ & $<0.001$ \\
\hline eGFR, per $1 \mathrm{~mL} / \mathrm{min} / 1.73 \mathrm{~m}^{2}$ greater & 0.96 & $0.95-0.97$ & $<0.001$ & 0.98 & $0.97-0.99$ & $<0.001$ \\
\hline Serum albumin, per $1 \mathrm{~g} / \mathrm{L}$ greater & 0.42 & $0.33-0.55$ & $<0.001$ & & & \\
\hline Serum IgA, per $1 \mathrm{~g} / \mathrm{L}$ greater & & & 0.177 & & & \\
\hline Gross hematuria (+) & & & 0.092 & & & \\
\hline Microscopic hematuria (+) & & & 0.608 & & & \\
\hline 24-hr urinary protein (> $1 \mathrm{~g} /$ day) & 4.21 & $2.70-6.56$ & $<0.001$ & 2.88 & $1.83-4.55$ & $<0.001$ \\
\hline HLA-DRB1*04 (+) & & & 0.268 & & & \\
\hline HLA-DQB1*04 (+) & & & 0.142 & & & \\
\hline HLA-DQB1*05:03 (+) & 0.58 & $0.33-1.02$ & 0.054 & & & \\
\hline
\end{tabular}

Abbreviations: IgAN, IgA nephropathy; Cr, creatinine; ESKD, end-stage kidney disease; eGFR, estimated glomerular filtration rate; HR, hazard ratio; Cl, confidence interval. 
Table 5. Associations of HLA with IgAN in various ethnic groups

\begin{tabular}{|c|c|c|c|c|}
\hline Ethnicity & Susceptible HLA allele & Protective HLA allele & Patients (N) & Reference \\
\hline \multicolumn{5}{|l|}{ Caucasian } \\
\hline French & DR4 & & 45 & Fauchet, et al. 1980 [7] \\
\hline British & DQ7 & & 36 & Li, et al. 1991 [24] \\
\hline French & $\mathrm{DRB}^{*} 04$ & & 58 & Raguénès, et al. 1995 [23] \\
\hline British & & $\mathrm{DQB1}{ }^{*} 02: 01$ & 105 & Fennessy, et al. 1996 [10] \\
\hline Finnish & & $\mathrm{DQB1}{ }^{*} 06: 02$ & 48 & \\
\hline \multicolumn{5}{|l|}{ Asian } \\
\hline \multirow[t]{2}{*}{ Japanese } & DR4 & & $24 / 42$ & Kashiwabara, et al. 1980 [8], 1982 [25] \\
\hline & B35, DR4, DQ4 & & $80 / 130 / 50$ & Hiki, et al. 1982 [9], 1990 [26], 1991 [27] \\
\hline \multirow[t]{4}{*}{ Chinese } & DRB1*14:05 & $\mathrm{DRB} 1 * 07: 01$ & 139 & Cao, et al. 2008 [28] \\
\hline & DRB1*04:05, *04:03 & & 935 & Jiyun, et al. 2012 [29] \\
\hline & $\mathrm{DQB1} 1 * 06: 01$ & $\mathrm{DQB1}{ }^{* 03: 01}$ & & \\
\hline & DRB1*0901-DQB1*06:01 & DRB1*07:01-DQB1*02:01 & 217 & Wang, et al. 2016 [30] \\
\hline \multirow[t]{3}{*}{ Korean } & $\mathrm{B} 55, \mathrm{DQB} 1 * 04$ & & 69 & Shin, et al. 1998 [31] \\
\hline & $\mathrm{DQB} 1 * 03$ & & 139 & Kim, et al. 2000 [14] \\
\hline & DRB1*04:05, DQB1*03:02, *04:01 & DRB1*07:01, *15:01, DQB1 ${ }^{*} 02: 02,{ }^{*} 06: 02$ & 399 & This study \\
\hline Caucasian+Asian & DQA1*01:01, DQB1*03:01 & $D Q A^{*} 01: 02, \mathrm{DQB} 1 * 02: 01$ & 2,747 & Kiryluk, et al. 2014* [32] \\
\hline
\end{tabular}

*HLA alleles investigated by imputation from GWAS data.

Abbreviations: HLA, human leukocyte antigen; IgAN, IgA nephropathy; GWAS, genome-wide association study.

kidney to his HLA-identical sibling with IgAN was observed to have occult IgAN in a biopsy, there have been many reports on associations between IgAN and HLA alleles [7-10, 14, 22-32] (Table 5). Studies on Japanese and French patients in the 1980s and 1990s reported that the HLA-DR4 allele frequency was significantly higher in IgAN patients [7-9, 23, 25].

We found associations between IgAN and HLA-DRB1*04:05, -DRB1*07:01, -DRB1*15:01, -DQB1*02:02, and -DQB1*06:02 in Korean patients. In China, the HLA-DRB1*04:05 and -DRB1* 04:03 allele frequencies were significantly higher in IgAN patients than in controls [29]. In the present study as well, the HLA-DRB $1{ }^{*}$ 04:05 allele frequency was significantly higher in IgAN patients than in controls. HLA-DRB1 ${ }^{*} 04: 05$ is associated with various autoimmune diseases, including rheumatoid arthritis and type 1 diabetes mellitus (T1DM) [33]. In particular, an association of HLA-DRB1*04:05 with a poor response to immunosuppressive therapy has been reported in Korean patients with aplastic anemia [34].

$\mathrm{HLA}-\mathrm{DQB} 1{ }^{*} 04$ is associated with IgAN in Japanese and Korean populations [27, 31]. However, the association of HLA$\mathrm{DQB1}{ }^{*} 04: 01$ with IgAN was newly identified in this study. HLADRB1 ${ }^{*} 04: 05$ and $-\mathrm{DQB1}{ }^{*}$ 04:01 exhibited a strong linkage disequilibrium (LD) (relative LD, 0.99) in a Korean population [16].
Therefore, the HLA-DRB1*04:05-DQB1*04:01 haplotype should be a strong susceptibility factor for IgAN in the Korean population, as has been reported for a Japanese population [27]. In addition, the HLA-DRB1*04:05-DQB1*04:01 haplotype is strongly associated with fulminant T1DM in Korean patients [35]. The HLA-DQB1*03:02 allele was newly revealed as a susceptibility factor for IgAN in this study.

Regarding protective alleles, HLA-DRB1*07:01 reportedly has a lower frequency in Chinese IgAN patients [28,30], which is in line with our results. HLA-DQB1*02:01 is reported to be a protective allele in British and Chinese IgAN patients [10, 30, 32]. However, in this study, $\mathrm{HLA}-\mathrm{DQB} 1{ }^{*} 02: 02$ was significantly lower in IgAN patients than in controls. The HLA-DRB1 ${ }^{*} 07: 01$ and -DQB1*02:02 alleles also exhibit strong LD (relative LD, 0.98) in the Korean population [16]. Therefore, HLA-DRB1*07:01 is suspected to play a major role as a protective factor, whereas HLADQB1 ${ }^{*} 02: 01$ or $-\mathrm{DQB} 1{ }^{*} 02: 02$ might be observed due to $\mathrm{LD}$ to HLA-DQB1*07:01, depending on ethnicity. HLA-DRB1*15:01 and $-\mathrm{DQB} 1{ }^{*} 06: 02$, which also show strong LD in the Korean population, showed lower frequencies in IgAN patients than in controls in this study. HLA-DQB $06: 02$ has been reported to be protective in Finnish IgAN patients [10]. The HLA-DRB1*15:01DQB1*06:02 haplotype reportedly protects against T1DM [36]. 
In Korean patients with aplastic anemia, HLA-DRB1*15:01 and $-D Q B 1 * 06: 02$ have been associated with a good response to immunosuppressive therapy, which suggests a possible role of HLA-DRB1*15:01-DQB1*06:02 in immune-related diseases [34].

The kidney survival rate of IgAN patients varies across studies, ranging from $57 \%$ to $91 \%$. Hypertension, decreased eGFR, and increased urinary protein at the time of diagnosis are wellknown risk factors for IgAN progression to ESKD [37]. In addition, lower plasma $\mathrm{Hb}$ concentrations and pathological results with higher Oxford $\mathrm{M}$ or $\mathrm{T}$ scores have been reported as risk factors for progression to ESKD [38]. Sex and age at diagnosis show different results in terms of posing risk of IgAN progression to ESKD among studies [37-39]. In our study, kidney survival was analyzed based on the presence of HLA-DRB $1{ }^{*} 04$, $-D Q B 1{ }^{*} 04$, and $-\mathrm{DQB1}{ }^{*}$ 05:03 alleles along with patient clinical and laboratory data; however, in the univariate analysis, none of these HLA alleles appeared as a risk factor for IgAN progression to ESKD. In the multivariate analysis, the levels of serum Cr, eGFR, and 24-hour urinary protein $>1 \mathrm{~g} /$ day were independent risk factors for IgAN progression to ESKD. Neither age nor sex was found to be a risk factor for IgAN progression to ESKD.

Regarding the association of HLA alleles with IgAN progression to ESKD, the allele frequencies of HLA-DRB1*04 and -DQB1 *04 were slightly, albeit not significantly, higher in the ESKD group than in the non-ESKD group. In the four-digit resolution analysis, the frequency of HLA-DQB1*05:03 was significantly lower in the ESKD group than in the non-ESKD group. However, the association of $\mathrm{HLA}-\mathrm{DQB} 1{ }^{*} 05: 03$ with IgAN progression to ESKD was not significant in Cox regression analysis, possibly due to the small number of patients and limited data available at diagnosis. Cao, et al. [28] reported that HLA-DRB1*03:01 is associated with decreased eGFR. In GWASs, SNVs related to the HLA region have been associated with IgAN progression to ESKD [32, 40]. The association of HLA alleles with IgAN progression to ESKD should be further studied in a larger patient cohort.

The limitations of this study are the relatively small number of IgAN patients with available clinical and laboratory data at diagnosis to analyze the risk factors for ESKD and the lack of pathological results (because the grading systems used for pathological diagnosis differed from patient to patient, depending on the time of diagnosis). However, our study showed that the HLADRB1 and HLA-DQB1 alleles are strongly associated with susceptibility to IgAN. While associations of HLA-DRB1*04:05, -DRB1*07:01, and -DQB1*06:02 alleles with IgAN have been previously reported in Chinese and Finnish patients, the associ- ations of the HLA-DRB1*15:01, -DQB1*02:02, -DQB1*03:02, and $-\mathrm{DQB1}{ }^{*} 04: 01$ alleles with IgAN in Korean patients were newly revealed in this study. Further studies on the association of HLA alleles with disease progression in a larger patient cohort and various ethnic groups are needed.

\section{ACKNOWLEDGMENTS}

The biospecimens used in this study were provided by the Biobank of Seoul National University Hospital, a member of Korea Biobank Network.

\section{AUTHOR CONTRIBUTIONS}

In JW performed experiments, collected and analyzed data, and wrote the manuscript. In JW, Lee H, and Song EY contributed to the study design. Jung $\mathrm{K}$ participated in collecting data. Shin S, Park KU, and Lee $\mathrm{H}$ reviewed the manuscript. Song EY participated in data analysis and manuscript writing. All authors read and approved the final manuscript.

\section{CONFLICTS OF INTEREST}

No potential conflicts of interest relevant to this study are reported.

\section{RESEARCH FUNDING}

This work was supported by the New Faculty Startup Fund from Seoul National University (800-20190499).

\section{ORCID}

Ji Won In

Kiwook Jung

Sue Shin

Kyoung Un Park

Hajeong Lee

Eun Young Song

https://orcid.org/0000-0001-8329-3512 https://orcid.org/0000-0001-8411-7473 https://orcid.org/0000-0003-4791-8671 https://orcid.org/0000-0002-2402-7633 https://orcid.org/0000-0002-1873-1587 https://orcid.org/0000-0003-1286-9611

\section{REFERENCES}

1. Schena FP and Nistor I. Epidemiology of IgA nephropathy: a global perspective. Semin Nephrol 2018;38:435-42.

2. Jennette JC, Wall SD, Wilkman AS. Low incidence of IgA nephropathy in blacks. Kidney Int 1985;28:944-50.

3. Barbour SJ, Cattran DC, Kim SJ, Levin A, Wald R, Hladunewich MA, et 
al. Individuals of Pacific Asian origin with IgA nephropathy have an increased risk of progression to end-stage renal disease. Kidney Int 2013; 84:1017-24.

4. Levy M. Multiplex families in IgA nephropathy. Contrib Nephrol 1993; 104:46-53

5. Suzuki H, Kiryluk K, Novak J, Moldoveanu Z, Herr AB, Renfrow MB, et al. The pathophysiology of IgA nephropathy. J Am Soc Nephrol 2011; 22:1795-803.

6. Kiryluk K and Novak J. The genetics and immunobiology of IgA nephropathy. J Clin Invest 2014;124:2325-32.

7. Fauchet R, Le Pogamp P, Genetet B, Chevet D, Guéguen M, Simon P, et al. HLA-DR4 antigen and IgA nephropathy. Tissue Antigens 1980;16: 405-10.

8. Kashwabara H, Shishido H, Yokoyama T, Miyajima T. HLA in IgA nephropathy. Tissue Antigens 1980;16:411-2.

9. Hiki Y, Kobayashi Y, Tateno S, Sada M, Kashiwagi N. Strong association of HLA-DR4 with benign IgA nephropathy. Nephron 1982;32:222-6.

10. Fennessy M, Hitman GA, Moore RH, Metcalfe K, Medcraft J, Sinico RA, et al. HLA-DQ gene polymorphism in primary IgA nephropathy in three European populations. Kidney Int 1996;49:477-80.

11. Gharavi AG, Kiryluk K, Choi M, Li Y, Hou P, Xie J, et al. Genome-wide association study identifies susceptibility loci for IgA nephropathy. Nat Genet 2011;43:321-7.

12. Feehally J, Farrall M, Boland A, Gale DP, Gut I, Heath S, et al. HLA has strongest association with IgA nephropathy in genome-wide analysis. J Am Soc Nephrol 2010;21:1791-7.

13. Yu XQ, Li M, Zhang H, Low HQ, Wei X, Wang JQ, et al. A genome-wide association study in Han Chinese identifies multiple susceptibility loci for IgA nephropathy. Nat Genet 2011;44:178-82.

14. Kim PK, Yook J, Kim JH, Jang YS, Shin JS, Choi IH. MHC class II allele association in Korean children with IgA nephropathy and its role as a prognostic factor. J Korean Soc Pediatr Nephrol 2000;4:33-9.

15. Jeong KH, Kim JS, Lee YH, Kim YG, Moon JY, Kim SK, et al. Genomewide association study identifies new susceptible loci of IgA nephropathy in Koreans. BMC Med Genomics 2019;12:122.

16. In JW, Roh EY, Oh S, Shin S, Park KU, Song EY. Allele and haplotype frequencies of human leukocyte antigen- $A,-B,-C,-D R B 1$, and -DQB1 from sequence-based DNA typing data in Koreans. Ann Lab Med 2015; 35:429-35.

17. Roh EY, In JW, Shin S, Yoon JH, Park KU, Song EY. Performance of LIFECODES HLA-DQB1 typing kit using Luminex platform in Koreans. Ann Lab Med 2015;35:123-7.

18. Nam M, Jung K, Roh EY, Shin S, Park KU, Song EY. Evaluation of LabType-SSO HLA typing for HLA-A, -B, -C, -DRB1, and -DQB1 loci. Clin Lab 2021;67:208-12.

19. Zetterquist $\mathrm{H}$ and Olerup $\mathrm{O}$. Identification of the HLA-DRB1*04, -DRB1*07, and -DRB1*09 alleles by PCR amplification with sequence-specific primers (PCR-SSP) in 2 hours. Hum Immunol 1992;34:64-74.

20. Spurkland A, Knutsen I, Markussen G, Vartdal F, Egeland T, Thorsby E. HLA matching of unrelated bone marrow transplant pairs: direct sequencing of in vitro amplified HLA-DRB1 and -DQB1 genes using magnetic beads as solid support. Tissue Antigens 1993;41:155-64.

21. Choe W, Chae JD, Yang JJ, Hwang SH, Choi SE, Oh HB. Identification of 8-digit HLA-A, -B, -C, and -DRB1 allele and haplotype frequencies in Koreans using the One Lambda AllType Next-Generation Sequencing Kit. Ann Lab Med 2021;41:310-7.
22. Tolkoff-Rubin NE, Cosimi AB, Fuller T, Rublin RH, Colvin RB. IgA nephropathy in HLA-identical siblings. Transplantation 1978;26:430-3.

23. Raguénès $\mathrm{O}$, Mercier $\mathrm{B}$, Clèdes J, Whebe $\mathrm{B}$, Férec $\mathrm{C}$. HLA class II typing and idiopathic IgA nephropathy (IgAN): DQB1*0301, a possible marker of unfavorable outcome. Tissue Antigens 1995;45:246-9.

24. Li PK, Burns AP, So AK, Pusey CD, Feehally J, Rees AJ. The DQw7 allele at the HLA-DQB locus is associated with susceptibility to IgA nephropathy in Caucasians. Kidney Int 1991;39:961-5.

25. Kashiwabara H, Shishido H, Tomura S, Tuchida H, Miyajima T. Strong association between IgA nephropathy and HLA-DR4 antigen. Kidney Int 1982;22:377-82.

26. Hiki Y, Kobayashi Y, Ookubo M, Kashiwagi N. The role of HLA-DR4 in the long-term prognosis of IgA nephropathy. Nephron 1990;54:264-5.

27. Hiki Y, Kobayashi Y, Ookubo M, Obata F, Kashiwagi N. Association of HLA-DQw4 with IgA nephropathy in the Japanese population. Nephron 1991;58:109-11.

28. Cao HX, Li M, Nie J, Wang W, Zhou SF, Yu XQ. Human leukocyte antigen DRB1 alleles predict risk and disease progression of immunoglobulin A nephropathy in Han Chinese. Am J Nephrol 2008;28:684-91.

29. Jiyun Y, Guisen L, Li Z, Yi S, Jicheng L, Fang L, et al. The genetic variants at the HLA-DRB1 gene are associated with primary IgA nephropathy in Han Chinese. BMC Med Genet 2012;13:33.

30. Wang W, Li M, Wang L, Yu X. DQB1*060101 may contribute to susceptibility to immunoglobulin A nephropathy in southern Han Chinese. Front Med 2016;10:507-16.

31. Shin YS, Kim YO, Park CW, Yoon SN, Yang CW, Kim YS, et al. Relationship between IgA nephropathy and histocompatibility antigens $(H L A)$ in Korean population. Korean J Med 1998;54:217-26.

32. Kiryluk K, Li Y, Scolari F, Sanna-Cherchi S, Choi M, Verbitsky M, et al. Discovery of new risk loci for IgA nephropathy implicates genes involved in immunity against intestinal pathogens. Nat Genet 2014;46:1187-96.

33. Arango MT, Perricone C, Kivity S, Cipriano E, Ceccarelli F, Valesini G, et al. HLA-DRB1 the notorious gene in the mosaic of autoimmunity. Immunol Res 2017;65:82-98.

34. Song EY, Kang HJ, Shin HY, Ahn HS, Kim I, Yoon SS, et al. Association of human leukocyte antigen class II alleles with response to immunosuppressive therapy in Korean aplastic anemia patients. Hum Immunol 2010;71:88-92.

35. Kwak SH, Kim YJ, Chae J, Lee CH, Han B, Kim JI, et al. Association of HLA genotype and fulminant type 1 diabetes in Koreans. Genomics Inform 2015;13:126-31.

36. Noble JA and Valdes AM. Genetics of the HLA region in the prediction of type 1 diabetes. Curr Diab Rep 2011;11:533-42.

37. D'Amico G. Natural history of idiopathic IgA nephropathy and factors predictive of disease outcome. Semin Nephrol 2004;24:179-96.

38. Xie J, Lv J, Wang W, Li G, Liu Z, Chen H, et al. Kidney failure risk prediction equations in IgA nephropathy: a multicenter risk assessment study in Chinese patients. Am J Kidney Dis 2018;72:371-80.

39. Hastings MC, Bursac Z, Julian BA, Villa Baca E, Featherston J, Woodford SY, et al. Life expectancy for patients from the Southeastern United States with IgA nephropathy. Kidney Int Rep 2017;3:99-104.

40. Wang W, Li G, Hong D, Zou Y, Fei D, Wang L. Replication of genomewide association study identified seven susceptibility genes, affirming the effect of rs2856717 on renal function and poor outcome of IgA nephropathy. Nephrol (Carlton) 2017;22:811-7. 